\title{
Recombinant Interleukin-3
}

National Cancer Institute

\section{Source}

National Cancer Institute. Recombinant Interleukin-3. NCI Thesaurus. Code C588.

A recombinant form of interleukin-3, a cytokine produced by activated T-cells and mast cells involved in intercellular communication, hematopoiesis, and inflammation. IL-3 binds and activates specific receptors on hematopoietic cells and in the nervous system, triggering expression of specific genes via the Ras signaling pathway and through Jak2 activation. This agent stimulates the proliferation of pluripotent hematopoietic progenitor cells. 